Horizons philosophiques

\title{
Le visage culturel de la mondialisation : un combat inégalitaire? Fondements philosophiques et perspectives légales
}

\section{Olivier Barré et Armelle Guignier}

Volume 15, numéro 2, printemps 2005

Cultures ou mondialisation?

URI : https://id.erudit.org/iderudit/801291ar

DOI : https://doi.org/10.7202/801291ar

Aller au sommaire du numéro

Éditeur(s)

Collège Édouard-Montpetit

ISSN

1181-9227 (imprimé)

1920-2954 (numérique)

Découvrir la revue

Citer cet article

Barré, O. \& Guignier, A. (2005). Le visage culturel de la mondialisation : un combat inégalitaire? Fondements philosophiques et perspectives légales. Horizons philosophiques, 15(2), 31-45. https://doi.org/10.7202/801291ar d'utilisation que vous pouvez consulter en ligne. 


\section{Le visage culturel de la mondialisation : un combat inégalitaire? Fondements philosophiques et perspectives légales}

II semblerait au regard de l'histoire, que les sociétés occidentales soient passées d'une gestion autarcique à une mondialisation des échanges économiques. Conjointement se sont tissés des liens culturels entre les nations. Nos identités culturelles actuelles sont issues de ce métissage qui jusqu'alors n'intéressait guère les marchés économiques ou alors qu'au titre de l'exotisme colonial. Le développement rapide de cette mondialisation, dû en partie à l'essor de technologie facilitant la communication, a permis l'augmentation du flux des échanges culturels mettant par là même ces derniers en relief, du moins beaucoup plus qu'ils ne l'étaient auparavant. Ainsi la culture est apparue au marché comme objet mercantile en puissance conformément à son héritage théorique. Cependant, cette objectivation de la culture en bien commercial, lorsqu'elle est mue par des politiques globalisantes, semble porter atteinte à l'existence des cultures issues de pays, voire de continents économiquement faibles. II apparaît alors que la culture du plus fort soit nécessairement la meilleure. Cette réalité met à mal nos propres conceptions intuitives de la culture qui place cette dernière dans un domaine universel de partage. Les impératifs mercantiles et les politiques globalisantes qui les sous-tendent sont néanmoins une réalité factuelle, qui, même si elle ne correspond pas à nos conceptions éthiques et morales normatives, doit être nécessairement envisagée comme telle. Dès lors notre propos ne va pas tant être de critiquer le processus global que de proposer une généalogie théorique de la mondialisation (I) afin de donner une assise conceptuelle à la perspective légale de la gestion et à l'encadrement par le droit international du bien culturel (II). 


\section{De l'héritage philosophique de la mondialisation à la} création d'un objet marchand

1) Quelques fondements théoriques

Le projet de vouloir identifier les facteurs explicatifs de la mondialisation, est somme toute, très ardu car il nécessite une transdisciplinarité. Nous allons nous borner, pour la suite, à mettre en évidence les éléments de philosophie politique et morale qui nous paraissent les plus pertinents afin d'expliquer a minima les fondements théoriques de la mondialisation. Cette dernière apparaît comme l'héritière de la pensée utilitariste couplée à la pensée libertarienne.

\section{a) La pensée utilitariste comme arrière-fond}

L'utilitarisme a pris son essor au cours des dix-septième et dix-huitième siècles en Angleterre par le biais d'une rupture de ses auteurs avec la tradition représentée par la morale aristotélicochrétienne ${ }^{1}$. Des auteurs tels que David Hume (17512), Jeremy Bentham (17893), John Stuart Mill (18614), ou encore Henry Sidgwick (18745) ont permis de donner corps, développement et diffusion à cette pensée. En effet, dans un contexte sociopolitique de questionnement de la légitimité des organisations sociales et des diversités d'allégeance à des systèmes moraux, ces penseurs utilitaristes ont permis de renouveler l'histoire de la pensée morale en ne fondant plus cette dernière sur les coutumes, les traditions ou encore le christianisme. Les décisions économiques et sociales se fondent désormais sur un principe fort simple : lorsque nous agissons, il faut que nous fassions abstraction de nos intérêts et de nos penchants, de nos préjugés et des tabous hérités de la tradition, ainsi que de tout prétendu «droit naturel», et que nous nous préoccupions exclusivement de poursuivre «le plus grand bonheur du plus grand nombre». II s'agit, en fait, de maximiser le bien-être collectif qui est analysé comme la somme du bien-être ou de l'utilité des individus composant la société observée. Lorsqu'il s'agit d'agir, l'utilitarisme exige que l'on établisse les conséquences associées aux divers choix possibles et que l'on évalue ensuite ces conséquences du point de vue de l'utilité des individus sujets, et qu'enfin l'on choisisse une des solutions possibles dont les conséquences soient telles que la somme des utilités individuelles qui lui est associée soit au moins aussi grande que celle associée à toute autre option possible. 
Mais, les ambitions normatives de l'utilitarisme et les hypothèses qu'elles mettent en avant, compliquent sévèrement la possibilité d'effectuer ce calcul. II s'ensuit tout au long du XXe siècle des querelles internes des penseurs utilitaristes afin de déterminer les conditions du calcul de maximisation. Ainsi les diverses critiques d'économistes de l'entre deux guerres ont conduit à l'hypothèse «ordinaliste», mais dès lors le principe premier de maximisation de l'utilité globale devenait inutilisable car il n'était plus possible de comparer les intensités de préférence entre individus. Le critère de "Pareto optimalité 6 » a semblé être une solution, cependant dans la réalité il arrive que des situations où les options entre lesquelles on doit choisir ne soient pas comparables ${ }^{7}$. II faut donc minimiser le risque d'incomparabilité, mais on observe que lorsque l'on tente de le faire, on maximise le risque d'irrationalité collective ${ }^{8}$. Dès lors, les utilitaristes doivent faire face à une faille épistémologique dans leur outil de calcul car "l'abandon du cardinalisme, (au profit de "l'ordinalisme») et par conséquent de la comparabilité interindividuelle des utilités, n'offre pas d'alternative idéale à la maximisation du bien-être agrégé ${ }^{9}$ ". Des auteurs, à l'exemple d'Arnsperger $^{10}$, tentent de proposer des solutions afin de répondre à ces problèmes de mesure. Cette approche philosophique de l'utilitarisme peut paraître loin des problématiques liées à la mondialisation cependant c'est bien sur un calcul utilitariste de maximisation que le système économique mondial repose. La mise en pratique de ce principe n'est pas représentative de la volonté générale de cette théorie. En effet aux prétentions premières de normativité ont suppléé des prétentions de rentabilité singulières, de la maximisation du bien collectif l'on passe à la maximisation du bien singulier.

b) Une pratique libertarienne

À la théorie utilitariste, il faut ajouter la pensée libertarienne pour comprendre la complexité du processus de mondialisation et le statut de la culture qui en découle. Ainsi, les principes de propriété de soi, de juste circulation et d'appropriation originelle s'additionnent au principe de maximisation des utilités.

Le premier principe est énoncé comme suit : «tout individu mentalement capable a un droit absolu à disposer de sa personne, y compris les talents qu'il a reçus et cultivés, pour autant qu'il n'utilise pas ce droit pour renoncer à sa propre liberté». Ce plein droit à disposer de soi-même détermine tout individu comme le plein propriétaire de son corps, de ses idées, et par extension de sa culture 
et il peut exercer un droit de veto sur ces derniers. De ce même fait, il peut également louer ses talents, exporter ses idées, et pourquoi pas, vendre sa culture. Bien qu'aucune restriction ne soit envisageable, les libertariens ne peuvent pas souscrire à l'esclavage et à l'intervention d'un tiers venant limiter leur principe fondamental. Cependant ce principe ne dit rien quant à l'usage qu'il s'impose moralement de faire de ce qui est en possession de chacun, et en cela le libertarisme se démarque de l'utilitarisme par les prétentions de ce dernier à fournir une théorie morale complète.

Dans le but d'user pleinement de la liberté conférée par ce premier principe, les libertariens ont ajouté un principe régissant la circulation des droits de propriété. La justice d'un droit de propriété est établie lorsque celui-ci a été obtenu par un transfert volontaire, tacite ou explicite, avec ou sans contrepartie matérielle ou monétaire, avec la personne qui en était auparavant propriétaire légitime. C'est donc sous la notion de "transaction volontaire" que les individus peuvent vendre ou acheter, par exemple, la culture de quelqu'un d'autre, mais lorsque les libertariens emploient ce terme ils y incluent l'exclusion de la fraude, de la violence physique et d'autres exactions (pressions économiques?).

Cette notion de transaction volontaire ne peut être appliquée que si l'on établit la légitimité du titulaire antérieur du droit de propriété. Le titulaire initial d'un droit de propriété sur un objet est le premier à en avoir revendiqué la propriété, éventuellement à condition de s'être acquitté d'une taxe dont le montant est fixé soit par la clause lockéenne (droit de tous à un sort au moins équivalent à ce qui aurait été dans l'état de nature), soit par le critère de justice painéen ${ }^{11}$ (droit égal de tous à la valeur des produits de la terre). Cette dernière limite à l'appropriation originelle, telle que Kirzner'12 l'a affirmée, tente de déterminer la légitimé d'une appropriation en fonction d'une taxe dont le montant reflète le montant des ressources dont le nouveau propriétaire s'arroge le droit de possession. La valeur de cette taxe est estimée en fonction des prix d'un marché pleinement concurrentiel et elle doit être redistribuée également entre tous. Ce libertarisme de gauche entraîne, outre le respect absolu de plein droit de propriété de chacun sur ses talents et sur les produits de ses talents, ainsi que la prohibition de toute réglementation des transactions volontaires, la création d'un impôt sur les biens de la transaction qui permettrait à chacun l'attribution d'un revenu de base. 
L'approche libertarienne ne peut être qualifiée ni de conséquentialiste, ni de configurationnelle, mais de strictement "historique», "rétrospective», ou encore "procédurale». Aux yeux d'un libertarien, il est en effet tout à fait impossible d'évaluer la justice d'une situation quelconque - en particulier d'une répartition des richesses ou des revenus - en spéculant sur ses conséquences ou en en analysant la structure. Pour déterminer si une situation est juste ou injuste, il faut et il suffit de se tourner vers le passé, d'en scruter le "pedigree", d'examiner si elle est le produit d'une procédure correcte ou d'un déroulement historique juste, c'est-à-dire d'un ensemble d'actions et de transactions effectuées dans le plein respect des droits affirmés par les trois principes ${ }^{13}$.

C'est donc dans un marché de libre concurrence s'appuyant sur un calcul utilitariste de maximisation et sur des principes libertariens de propriété que la culture est jetée en pâture aux plus riches des entrepreneurs mondiaux. Cette finalité économique concurrentielle rejetant toute considération morale en terme de conséquence de cette politique menée, nous paraît très loin d'ụn esprit humaniste qui se représente intuitivement la culture comme un donné universel inaliénable dans un patrimoine mondial.

\section{2) Quand la culture devient objet de commerce}

Dès lors, comment comprendre le processus par lequel la culture est passée d'un support sur lequel l'activité commerciale transitait à l'objet même de cette activité. Comment s'est opérée cette mutation d'une caractéristique nationale ou ethnique, à un objet singulier de commerce? L'une des réponses possibles serait à chercher, sans doute, dans la nécessité que le marché a de trouver toujours de nouveaux objets de commerce afin de se régénérer. Cependant, il serait également intéressant de tourner les yeux vers les politiques économiques nationales afin de considérer ces dernières comme étant encore dans une dynamique post-colonialiste, ce qui nous amène à penser, en terme de finalité utilitariste, que les états les plus puissants ont toutes utilités à vouloir imposer leur propre culture. Partant de ce constat, ce n'est pas sur une culture réelle dans les faits que vont s'appuyer les entrepreneurs pour proposer à la vente leur bien culturel, mais bien plus sur une construction idéale, en ce qu'elle peut représenter de meilleur. 
a) La création et la vente de l'idéal culturel

Ce bien culturel est bien plus que ce qu'il est. À travers lui, c'est l'image, la représentation idéale d'un monde qui véhicule des idées de liberté, de force, d'action. Quand un bien culturel venant d'un pays étranger est acheté, c'est certes l'objet en lui-même qui est acheté mais c'est aussi le «packaging culturel» qu'il représente. Ainsi petit à petit les biens culturels étrangers tendent à détourner le consommateur de sa propre identité culturelle voire même à l'en déposséder.

Cet idéal érigé en proue par des campagnes publicitaires offensives témoigne des politiques marketing des entreprises qui construisent cette image idyllique qu'une culture économiquement forte peut créer. Ainsi, c'est la culture du plus fort qui est toujours la meilleure.

II y a quelque chose de pernicieux dans cette mécanique de la représentation d'un idéal culturel. En effet, s'il y a des critiques sur l'intolérance culturelle qu'une telle politique peut engendrer, cette machinerie s'adapte en arguant du fait que dans 'l'idéal proposé est inclus le plus profond respect des cultures locales. II n'empêche que dans la pratique, une fois ces biens culturels mis sur le marché, plus aucune considération n'est faite des cultures locales, ces dernières rentrent nécessairement dans le champ de la libre concurrence, sans s'interroger sur une quelconque égalité des moyens de concurrence.

b)Le décalage entre la réalité d'une culture et ses objectifs mercantiles. Le cas des États-Unis.

Les États-Unis n'en seront pas à une contradiction près, à ceci de particulier que cette dernière semble d'une rare énormité. En effet, au pays de la libre entreprise et de la liberté individuelle, l'identité des minorités et les cultures qui s'y rattachent sont préservées par des lois de discrimination positive qui permettent à tous ceux qui le souhaitent de pouvoir exprimer librement leur différence ethnique et culturelle. L'espace de la politique intérieure du melting pot permet l'émergence d'une considération singulière, ne fût-elle que réglementaire, d'une nécessité à reconnaître et à donner une place aux minorités. Par extension n'y aurait-il pas par là, la voie d'une reconnaissance tacite de la nécessité d'un pluralisme culturel actif au sein même d'un pays qui a élaboré, pendant plus de cinquante années, l'image de sa culture sur le White Anglo-Saxon Protestant?

Comment comprendre que s'agissant de politique intérieure une sorte de protectionnisme culturel en faveur des minorités soit appliqué 
et que dès qu'il s'agit de politique économique extérieure l'identité culturelle de pays dits "en voie de développement" ou encore de pays à forte identité culturelle et ayant les moyens de la revendiquer, soit la proie des attaques incessantes de ce néo-impérialisme qui ne souhaite que prendre leurs places? Comment saisir la cohérence? La seule compréhension que nous pourrions apporter semble tenir au réalisme qui voit «chez l'autre» le moyen d'accroître son influence et ses biens mais qui ne tolère pas qu'il en soit fait de même à lui-même. Ainsi, nous rompons ici avec l'impératif kantien qui demande à celui qui agit, s'il agirait de la même façon si c'était lui qui devait en subir les conséquences. Bref, l'autonomie kantienne est à cent mille lieux des interrogations des parties agissant en fonction de leur propre intérêt. L'unique objectif : l'exportation culturelle afin de maximiser les utilités particulières et tout cela sans une once de moralité. J'aimerais soulager ceux qui seraient amenés à taxer mes propos, à travers l'exemple pris, d'anti-américanisme primaire, par le fait que, d'une certaine façon la critique faite à l'encontre des États-Unis peut être faite également, sous certains aspects, à la politique de la francophonie menée par la France.

Une fois de plus, il nous semble que le rapport mondialisation/ culture ne pose réellement problème qu'aux États occidentaux, du moins lorsqu'il est posé sur les bases de rapports économiques, d'influence, de pouvoir. Une guerre ouverte et stigmatisée en 1996 par les non- accords du GATT sur les biens culturels plante un décor de bipolarité entre les États-Unis et l'Europe. Guère de place semble-t-il pour les pays du Sud qui doivent gérer des situations et des problèmes de nature différente portant pour certains sur l'unique survie. Nous aurons compris que lorsque l'on a faim, les revendications culturelles sont mises sous éteignoir. Cependant, il nous semble urgent d'établir une réglementation appropriée afin de permettre aux pays en voie de développement, de préserver dès maintenant ce qui fait et fera leur force culturelle de demain contre les attaques des nations économiquement dominantes. La question reste en suspens : quels moyens et solutions pour un rééquilibrage international de la concurrence culturelle?

\section{Les réponses du droit : protection des cultures contre la mondialisation ou concurrence équitable entre cultures?}

La mondialisation est ambivalente : elle peut être source d'interactions entre les cultures mais également source de risques d'appau- 
vrissement, voire d'homogénéisation ${ }^{14}$. Dès lors, le droit international peut-il apporter une réponse à ce double défi ainsi créé?

D'une part, comment le droit peut-il protéger la culture en tant que valeur non marchande face à la mondialisation économique, et d'autre part, comment peut-il contribuer à garantir les conditions d'égalité des chances des cultures mises en concurrence par ce marché mondialisé?

1) La place de la culture dans la mondialisation économique

a) La culture dans le droit international économique

Au titre de régulation des relations internationales au niveau mondial, le droit international constitue un moyen. Or le droit est également touché de plein fouet par la mondialisation. La mondialisation économique se traduit notamment par un droit international économique hégémonique prenant la forme des accords de l'Organisation Mondiale du Commerce. C'est autour de ce droit économique que la question de la culture s'est cristallisée sur la scène internationale. Ainsi, la culture est entrée dans la compétition propre au marché.

Depuis la création de l'OMC, le droit international économique s'est étendu aux biens et services culturels en ne posant aucune exception culturelle dans les accords relatifs aux services et aux biens. La culture ne peut échapper au marché démontrant l'aspiration irrésistible de nombreuses valeurs non marchandes dans le système commercial. En effet, elle est perçue comme un frein au libéralisme car certains États entendent la faire échapper au commerce en adoptant des attitudes protectionnistes.

En quelques mots, les services culturels ne sont pas exclus de l'Accord Général sur les services (AGSC), il n'existe aucune exception culturelle dans l'accord de l'OMC. En effet, l'AGSC s'applique à tous les services pour autant qu'ils soient marchands (à l'exception "des services fournis dans l'exercice du pouvoir gouvernemental» art.I.3.b). II n'y a pas d'exclusion culturelle comme dans l'Accord de libre-échange nord américain (ALENA) ${ }^{15}$.

Ainsi peuvent-ils être soumis aux principes traditionnels de I'OMC : égalité de traitement et non discrimination.... Cependant, il appartient à chaque membre de l'OMC de souscrire des engagements spécifiques de libéralisation de certains secteurs (concernant l'accès au marché et le principe du traitement national), mais ils ont également la possibilité d'exempter certains secteurs de l'application 
de la clause de la nation la plus favorisée par dérogation au principe général lors de l'acceptation de l'AGSC'16.

Quant aux biens culturels, ils sont soumis aux principes de l'Accord sur le commerce des marchandises (GATT) à l'exception des films cinématographiques ${ }^{17}$. Le régime juridique est beaucoup moins souple que pour l'AGSC, car tous les principes libéraux s'appliquent.

b) La culture, "une marchandise pas comme les autres"

II apparaît clairement que la culture, sous sa forme de biens et de services culturels, se trouve affectée par ce droit économique libéral. La culture illustre comme l'environnement ou les droits de l'homme, les conflits de valeurs qui surgissent dans cette mondialisation économique. Les biens et services culturels ont une double dimension : l'une commerciale, l'autre culturelle qui n'est pas intégrée par le commerce. Or, aujourd'hui, les piliers du développement durable ne peuvent se limiter à l'environnement, à l'économique et au social. Le domaine culturel apparaît comme un $4^{e}$ pilier légitime. Dès lors, l'intégration de la culture comme valeur non marchande doit également se réaliser dans le monde économique, afin que les aspects immatériels de la culture soient pris en compte indépendamment des moyens matériels et marchands par lesquels elle est véhiculée.

On ne peut pas parler de culture dans un sens restrictif. La culture englobe certes les films, les livres (ce qui pourrait rentrer dans le cadre de biens et services culturels pouvant faire l'objet d'un commerce), mais la culture est représentative également des valeurs attachées à une société, un État ${ }^{18}$. Cela peut concerner l'alimentation mais également les langues, le droit. Ces valeurs sont toutes affectées plus ou moins par la mondialisation : mondialișation économique, juridique, culturelle. Si le commerce constitue l'un des véhicules d'idées, de biens et de services culturels, d'autres facteurs non marchands permettent de transmettre une culture à une autre culture (éducation, télécommunications, politique...)

II apparaît d'autant plus important d'envisager les aspects non marchands de la culture que la mondialisation s'inscrit dans le cadre de "stratégies transnationales à caractère privé». Ces dernières ont un impact sur tous les échanges y compris culturels ${ }^{19}$, affectant ainsi la souveraineté des États et rendant la protection culturelle plus fragile. Cette position montre alors que les intérêts immédiats, mercantiles sont privilégiés au détriment de finalités plus durables et non commerciales. 
2) La concurrence culturelle face à la diversité culturelle

Deux tendances complémentaires semblent se dégager : d'une part, la protection directe de la diversité culturelle par des textes appropriés, et d'autre part, la nécessité d'établir des conditions adéquates pour rendre équitable l'incontournable concurrence entre les cultures, en d'autres termes donner des chances égales à la création culturelle, à la circulation des cultures, à l'accès des populations à cette diversité culturelle. Quels moyens doivent être mis en place pour diminuer les déséquilibres d'accès, de diffusion, de réception, de transmission de la diversité culturelle?

a) La protection de la diversité; culturelle : nouveau défi du droit

La diversité culturelle est aujourd'hui considérée comme patrimoine commun de l'humanité20. En d'autres termes, elle serait exclue de la souveraineté des États, ne pourrait faire l'objet d'aucune appropriation ${ }^{21}$ et devrait faire l'objet d'une solidarité entre générations présentes mais également envers les générations futures.

La diversité culturelle devient un objet de droit. Des outils et des mécanismes tendant à assurer sa protection se mettent en place.

L'État doit-il conserver un pouvoir d'intervention en matière culturelle afin de protéger sa diversité culturelle? Des mesures restrictives au commerce des biens culturels devraient être possibles si le commerce est susceptible de porter atteinte à la diversité culturelle, mais les échanges de biens et services culturels doivent également être encouragés car source d'échanges culturels. Pour certains États, la meilleure protection de la culture passe par l'exclusion des biens et services culturels du libéralisme. Ainsi, les ministres de la culture de l'organisation internationale de la francophonie ont-ils déclaré que «la façon de préserver la diversité culturelle demeure de s'abstenir de prendre des engagements de libéralisation en matière de biens et services culturels", lors de la Déclaration de Cotonou du 15 juin 2001 (point 9\$3).

Cette approche défensive passant par l'exception culturelle est aujourd'hui en recul, et fait place à une approche offensive ${ }^{22}$ pour une protection de la diversité culturelle et une amélioration des échanges culturels favorisant celle-ci. Afin de protéger la diversité culturelle, les organisations internationales se sont impliquées dans l'élaboration de textes non contraignants (Conseil de l'Europe, Union européenne, UNESCO) et en particulier la Déclaration universelle sur la diversité culturelle. Mais l'innovation proviendra dans les années à venir de 
l'adoption d'un acte contraignant dénommé Convention sur la protection des contenus culturels et des expressions artistiques. À l'heure actuelle, ce document figure à l'état de projet et a suscité de nombreuses interrogations quant à sa compatibilité avec les accords de l'OMC23. L'un des droits qui découlera de cette convention sera la reconnaissance du «droit souverain des Etats d'adopter des mesures pour protéger et promouvoir la diversité des expressions culturelles sur leur territoire, et leur obligation de la protéger et de la promouvoir sur leur territoire et à l'échelle mondiale 24 ", tout en respectant leurs obligations découlant d'autres accords internationaux, dont l'OMC.

Cela ne résout qu'une partie du problème car cette convention pourra-t-elle remédier aux déséquilibres actuels que subissent les cultures ou ne faudra-t-il pas introduire dans le cadre des accords de I'OMC, des clauses garantissant l'établissement d'une égalité entre cultures?

b) Poser l'égalité des chances entre cultures:

On peut se demander si l'idéologie libérale ne véhiculerait pas une culture uniforme dans le sens où les États doivent adapter leur système en fonction des exigences libérales de l'OMC? Certes, les États souverains ont adhéré volontairement aux accords (et majoritairement des pays en développement) et à la philosophie de ceux-ci, mais il ne faut pas oublier que leur souveraineté s'attache également à décider de leur modèle politique, culturel, ou de développement. Cette souveraineté leur permet également, mais selon des exigences strictes, de se soustraire à leurs obligations (ex : santé publique, sécurité, environnement...).

Quelle marge de manœuvre existe alors pour permettre aux États non seulement de protéger les différents aspects culturels de leur société mais également de diffuser leur culture sur un pied d'égalité malgré la propagation massive d'autres cultures ayant bénéficié du libre-échange?

Les déséquilibres des échanges de biens et services culturels sont réels, services et biens culturels entrent en concurrence sur un marché mondialisé dans une situation d'inégalités. Dès lors, comment poser les conditions d'égalité? D'un point de vue purement théorique (ex : thèse d'Hayek), l'ordre spontané du marché reviendrait à tout libéraliser et à poser ainsi d'égales conditions pour tous. Cependant, cette thèse ne prend pas en compte les risques de domination économique de certains États qui se détournent des principes 
libéraux, voire les récusent dans leurs intérêts ${ }^{25}$. Le libéralisme permettrait de mettre en concurrence parfaite les services et les biens culturels. Cependant, comment permettre à un petit État du Sud d'avoir des chances égales de faire percer sur un marché européen ses propres biens et services culturels?

On ne peut pas ainsi résumer et résoudre la question de la concurrence entre cultures d'un point de vue exclusivement économique, et dans des termes de facilitation d'accès des biens et services sur le marché mondial même si cette démarche est privilégiée notamment par la coopération au développement. En témoigne l'accord de Cotonou entre l'Union européenne et les pays Afrique-Caraïbes-Pacifique qui aborde à la fois la protection de la culture mais également l'amélioration des conditions d'accès au marché des biens et services culturels ${ }^{26}$. Cependant, cette dernière solution n'est pas suffisante en elle-même pour garantir l'égalité des chances entre culture des États du sud et culture occidentale par exemple, d'autant plus que les pratiques anticoncurrentielles des entreprises ne font pas encore l'objet d'un texte spécifique à l'OMC relatif à la concurrence 27 , renforçant ainsi les situations d'inégalités.

L'égalité ne passe-t-elle par la préservation de la liberté culturelle et du droit d'accès des individus pour lesquels les États ne peuvent adopter une attitude protectionniste qui limiterait leurs choix? Pour assurer la survie de la diversité culturelle, le libéralisme n'est pas la solution ni le repli protectionniste.

L'égalité doit-elle passer par l'inégalité? Doit-on voir se mettre en place des quotas internationaux pour les produits culturels en provenance des États du Sud? La solution ne réside sans doute pas dans cette discrimination positive, mais les États du nord doivent reconnaître la responsabilité du libéralisme dans ces déséquilibres (que cela soit en terme d'échanges de biens et services culturels, mais également et plus largement, de l'impact économique du libéralisme qui ne tend pas à aider les États du Sud à investir dans la création culturelle faute de moyens).

Dès lors, les États plus développés ne devraient-ils pas restreindre leur propre liberté d'action? Le projet de convention parle d'un traitement préférentiel accordé par les pays développés aux pays en développement afin de favoriser les échanges culturels. Cependant, les questions des politiques "d'attaques culturelles" ne sont pas envisagées, or les États développés en limitant leurs politiques qui bénéficient du libre-échange et de l'ouverture de frontières des États 
du Sud, pourraient au contraire contribuer au bénéfice des cultures dans les États du Sud, sur la base du respect de ces cultures. À terme, les États du Sud, moins efficaces sur le marché que les pays du Nord, seront absorbés par une culture, celle qui profite du libre-échange, faisant ainsi disparaître les potentialités de création culturelle et l'appauvrissement de la diversité culturelle que l'on entend protéger pour les générations futures.

Les théories philosophiques utilitariste et libertarienne contribuent à la compréhension du droit actuel de l'économie internationale. Le libéralisme, tel que l'énoncent les premiers mots de la déclaration ministérielle de Doha ${ }^{28}$, conduit à en faire le seul et unique moteur du développement. Deux problèmes apparaissent alors. D'une part, les valeurs non marchandes semblent les parents pauvres du droit international économique. La culture n'est envisagée que sous son visage de "bien marchand", et la nécessité de faire contrepoids à l'idéologie marchande apparaît de plus en plus, à l'exemple du projet d'instrument juridique en faveur de la diversité culturelle. D'autre part, l'objet mercantile qu'est devenue la culture conduit à une inégalité mondiale des cultures en raison des rapports de force économique par lesquels elle est véhiculée. C'est bien l'absence d'égalité des chances en terme de concurrence qui est au cœur de ce problème. Même si notre objectif premier n'était pas de proposer une alternative globale au système en place, il n'en demeure pas moins que l'ensemble de cette étude, de par son approche pluridisciplinaire : philosophique et juridique, pose la nécessité de rapports équitables, et pas uniquement égalitaires, entre les cultures dominées et dominantes afin que l'équilibre puisse s'opérer. Cependant les modalités d'une telle équité restent à débattre.

\author{
Olivier Barré \\ DEA en philosophie, \\ Faculté des Sciences Humaines, \\ Université Toulouse le Mirail ef \\ Université Bordeaux III, France.
}
Armelle Guignier
Doctorante en droit, Centre de Recherche interdisciplinaire en Droit de l'Environnement, de l'Aménagement et de l'Urbanisme, CRIDEAU-CNRS-INRA, Faculté de Droit et Sciences économiques, Université de Limoges, France. 
1. Nous nous inscrivons ici dans la tradition de l'histoire de la pensée pour évoquer I'histoire de ce courant. Cependant il est à noter qu'il existe une autre lecture de I'histoire de l'utilitarisme, à travers l'interprétation issue du Groupe de MAUSS (Mouvement Anti-Utilitariste dans les Sciences Sociales) qui fait remonter le principe d'utilité ou du bonheur aux philosophes grecs (Platon, Aristote, etc.) ainsi qu'aux pères de l'Église et notamment st Augustin.

2. David Hume, Enquêtes sur les principes de la morale (1751), traduction, préface et notes d'André Leroy, Paris, Aubier, 1947.

3. Jeremy Bentham, An Introduction to the Principles of Morals and Legislation (1789), in J.S. Mill, Utilitarianism, Fontana, Londres, 1962. On peut également le retrouver édité par J.H. Burns et H.L.A Hart, Methuen, London and New York, 1982.

4. John Stuart Mill, Utilitarianism, Londres, Fontana, 1962. La traduction française : L'Utilitarisme, Paris, Flammarion, 1988.

5. Henry Sidgwick, The Methods of Ethics, Londres, Macmillan, 1874.

6. «Une situation A est Pareto optimale s'il n'existe aucune autre situation possible B telle que, d'une part, un individu au moins préfère strictement $B$ à $A$, tandis que, d'autre part, aucun individu ne préfère $A$ à $B$. Une implication directe est que, si $A$ est Pareto optimal, il n'existe pas d'autre option qui lui soit unanimement préférée". Arnsperger et Van Parijs, Éthique économique et sociale, Paris, La Découverte, coll. «Repères», 2003, p. 21.

7. Cf., théorème d'impossibilité d'Arrow.

8. Nous renvoyons ici au paradoxe de Condorcet.

9. Arnsperger et Van Parijs, Éthique économique et sociale, Paris, La Découverte, coll. «Repères", 2003, p. 23.

10. Christian Arnsperger, Modemity and the Strange Demise of Utilitarianism, Fribourg, Universtätsverlag Freiburg, in B. Sitter-Liver et P. Caroni (sous la dir. De), Der Mensch - Ein Egoist?, 1998, p. 23-40

11. En référence à Thomas PAINE dans, Agrarian Justice, en 1796, traduit en français : La justice agraire, dans le Bulletin du MAUSS, vol. 7, n 1, en 1996.

12. Israël M. Kirzner, Perception, Opportunity, and Profit. Studies in the Theory of entrepreneurship, Chicago, University of Chicago Press, 1979.

13. Arnsperger et Van Parijs, op. cit., p. 38.

14. Préambule avant-projet de Convention sur la protection des contenus culturels et des expressions artistiques, Paris, UNESCO, CLT/CPD/2004/CONF.201/2, juillet 2004.

15. Les industries culturelles sont exemptées des dispositions de l'ALENA.

16. Ce fut le cas pour le secteur audiovisuel de la Communauté européenne. Cette dernière a décidé de ne pas souscrire d'engagement de libéralisation pour son secteur audiovisuel, et donc de le faire échapper aux principes libre-échangistes.

17. L'article IV du GATT permet des contingents pour les films d'origine nationale.

18. Art. 4 avant-projet de Convention définit la culture comme «l'ensemble des traits distinctifs spirituels et matériels, intellectuels et affectifs qui caractérisent une société ou un groupe social et englobe, outre les arts et les lettres, les modes de vie, les façons de vivre ensemble, les systèmes de valeurs, les traditions et les croyances". 
19. Mireille Delmas-Marty, Les forces imaginantes du droit : le Relatif et I'Universel, Paris, Seuil, p. 36.

20. Art. 1 Déclaration universelle sur la diversité culturelle, UNESCO, 2 novembre 2001.

21. Agnès Lejbowicz, Philosophie du droit international. L'impossible capture de I'humanité, Paris, PUF, 1999, p.165.

22. Thomas Paris, "Diversité culturelle et mondialisation", p.184 in RAMSES, Rapport annuel mondial sur le système économique et les stratégiest sous la direction de Thierry de Montbrial et Philippe Moreau Defarges, IFRI, 2004.

23. Ivan Bernier, Hélène Ruiz-Fabri, Evaluation of the Legal Feasability of an International Instrument Governing Cultural Diversity, Groupe de Travail Francoquébécois sur la diversité culturelle, Bibliothèque nationale du Québec, 2002, 50 p.

24. Art. 5 avant-projet de Convention, op.cit.

25. OST François, «Mondialisation, Globalisation, universalisation : s'arracher encore et toujours à l'état de nature", p. 11, in Le droit saisi par la mondialisation sous la direction de Charles-Albert Morand, Bruxelles, Bruylant, 2001.

26. Art. 27, Accord de partenariat en Union Européenne et pays ACP, Cotonou, 20 juin 2000, entré en vigueur $1^{\mathrm{er}}$ avril 2003.

27. L'OMC s'est attachée à la libéralisation des échanges sans mettre en place un droit international de la concurrence pourtant complémentaire. Thiébaut Flory, L'organisation mondiale du commerce :Droit institutionnel et substantiel, Bruxelles, Bruylant, 1999, p. 211.

28. Déclaration Ministérielle adoptée le 14 novembre 2001, Conférence ministérielle, Quatrième session, Doha, WT/MIN(01)/DEC/1, 20 novembre 2001, p. 